\title{
Effect of heat and drought stress on the expression of regulatory transcription factors and the genes involved in different metabolic pathways
}

Murali Oleti

Department of Botany, College of Sciences, Sri Venkateswara University, Tirupati, Andhra Pradesh, India.

Received: 04-12-2017; Revised: 11-12-2017; Accepted: 27-12-2017

\begin{abstract}
Plants never encounter stress factors, whether biotic or abiotic in sequence. Mostly they have to face multiple environmental factors in suboptimal level (stressful) at the same time. As a result, the strategy of the plant to survive in such situations demands handling of multiple stresses at the same time by efficient management of the genetic repertoire that the plant has. The plant achieves this by altering expression of transcription factors that regulate the activity of different genes, whose products themselves play the structural and functional roles. In the present study, differentially regulated genes under heat and drought stress from different microarray studies were analyzed to assess the kind of metabolic pathways that are specifically altered (promoted or sacrificed) under heat and drought stress and the transcription factor families which have the governing role in such regulatory processes. It was observed that expression of genes related to metabolism, specifically in the cell organelles like plastids and mitochondria is differentially regulated. Some transcription factor families like AP2-EREBP, NAC, C2H2 and MYB play more important role in the two kinds of stress conditions.
\end{abstract}

Keywords: Heat stress, Drought stress, transcription factor, Gene regulation.

\section{Introduction}

During its life time, a plant rarely if at all, experiences the environment in optimum condition. At every instance one or the other factor fluctuates from optimal to suboptimal (stressful) level. The plant has to constantly respond to the changing environment by altering its metabolic repertoire, to survive in the extant environmental conditions. For this the plant senses the environmental signals and alters the level of its transcripts accordingly. Two such stress factors, heat and drought, mostly affect the plant together or in very close sequence in desertic conditions. To counter the effects of stress, plants undergo a process of stress acclimation. This process may require changes in the flow of metabolites through different pathways, the suppression of pathways that may be involved in the production of ROI during stress, and the induction of various defense genes (Xiong et al., 1999; Dat et al., 2000; Mittler, 2002). It has been shown that, different stress conditions such as drought and cold result in the activation of similar stress response pathways (Seki et al., 2001; Chen et al., 2002). This indicates a high degree of overlap may exist between gene clusters activated by different stresses. This overlap explains the welldocumented phenomena of "cross tolerance," in which a particular stress can induce in plants resistance to a subsequent stress that is different from the initial one (Bowler and Fluhr, 2000).

\section{${ }^{*}$ Corresponding Author:}

\section{Dr. Murali Oleti,}

Department of Botany,

College of Sciences, Sri Venkateswara University,

Tirupati, Andhra Pradesh, India.

E-mail: murali.oleti@gmail.com
Study of abiotic stress response has advanced enormously in recent years. In contrast to classical genetic approaches, where one or a few genes could be studied at a time, with the development of microarray technology and its advancements, it has become possible to study the effect of a particular factor on the gene expression of an organism. This technology heavily depends on the development of computing efficiency from the stage of setting up the experiment, analysis of the image to analysis of the result. The present study takes advantage of the resources available from microarray studies and attempts to assess the effect of drought and heat stress on the relative expression of genes. This study aims at identifying the common pathways utilized by the plant under both kinds of stresses.

\section{Materials and Methods}

Microarray experiments are used to analyze the effect of specific treatments on the expression of the entire genome. The genes differentially expressed (significantly up or down regulated), can then be statistically distinguished to understand the effect of the treatment on different metabolic pathways. In this study, gene list of differentially regulated genes under heat and drought stress were retrieved from three different studies (Charng et al., 2006; Abdeen et al., 2010; Mizoguchi et al., 2010). The functional annotations of the different genes and the information about the different pathways in 
which they are specifically involved were retrieved from the FunCatDB (Mewes et al., 2010).

\section{Results and Discussion}

Information about differential expression for 4008 genes was downloaded. Of these 208 were significantly upregulated, and 196 significantly downregulated under heat stress. Similarly, 1706 genes were significantly upregulated, and 1908 genes were significantly downregulated under drought stress.

Under heat stress the number of upregulated genes involved in different processes ranged from 1 involved in regulation of protein activity to 74 , in specific processes in different compartments of the cell. Out of the 74 genes, 41 were found to be involved in process in the mitochondria.

Under heat shock a total of 196 genes were downregulated, and their numbers according to functional category ranged from a single gene involved in cell type differentiation, to 68 genes involved in different organelles of the cells. The processes which were found to be severely affected under heat shock due to down regulation of the genes included cell development (5 genes), cell cycle and DNA processing (7 genes), biogenesis of cellular components (12 genes), transcription (14 genes), cellular communication and signal transduction (19 genes, Fig. 2).
Under drought stress, a total of 1706 gene were upregulated, of these, only 2 were involved in tissue differentiation, while the maximum number of genes (412) were related to specific functions in different organelles of the cell (Fig.3). These genes were involved in cellular functions in different compartments of cell like, 80 genes were involved in nuclear functions, 153 in mitochondria and 138 in plastids.

Maximum number of genes (1908) were significantly downregulated under drought stress. The number of genes downregulated under drought stress, ranged from 3 (storage protein genes), to 539 genes (in specific processes in different specialized compartments of the cell). 419 genes, with reduced transcription, were responsible for producing proteins with binding function or cofactor requirement (Fig. 4).

When analysis of the transcription factor genes up or downregulated under a specific type of stress was carried out, it was noticed that there were substantial differences in the transcription factor family and the number of genes specifically up or down regulated under a particular stress. Out of the 50 different families of transcription factors (TFs) catalogued in the AGRIS database (Yilmaz et al., 2011); in the present analysis 32 families were represented. The expression of TFs was more influenced by drought stress (91 up and 86 downregulated), as compared to heat stress (14 up and 14 downregulated). The families that had more genes significantly upregulated under drought stress were AP2-EREBP and NAC (Fig. 5).

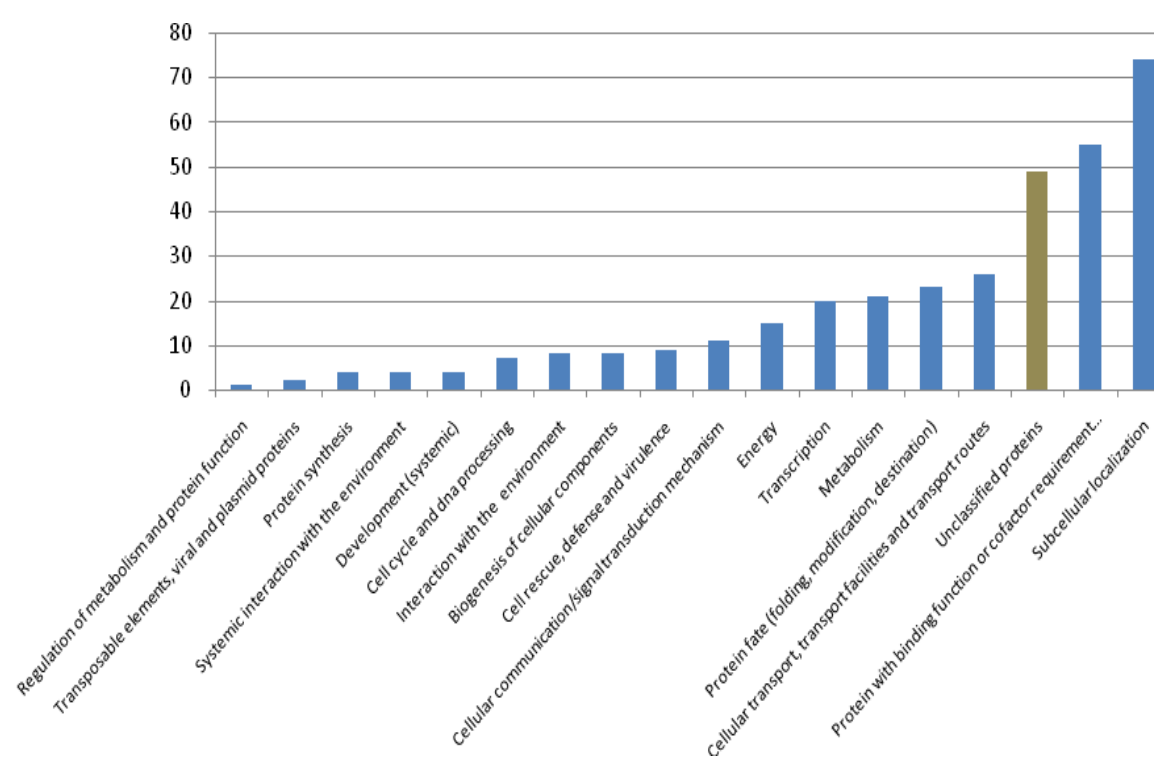

Figure 1. Genes involved in different metabolic pathways significantly up regulated under heat stress 


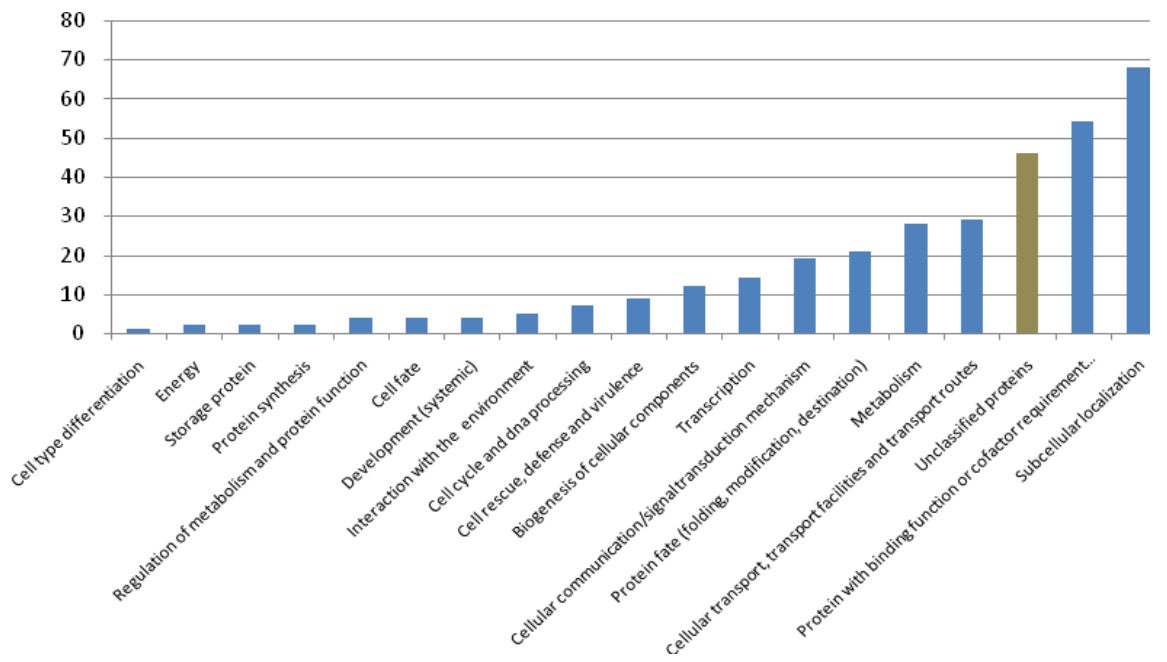

Figure 2. Genes involved in different metabolic pathways significantly down regulated under heat stress

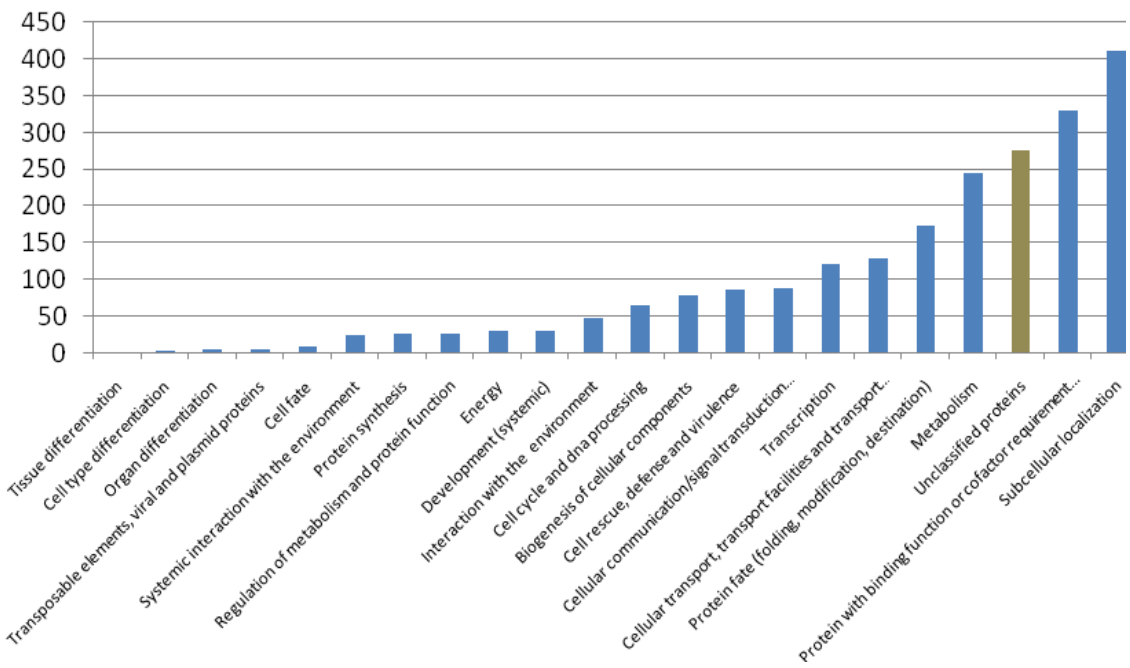

Figure 3. Genes involved in different metabolic pathways significantly up regulated under drought stress

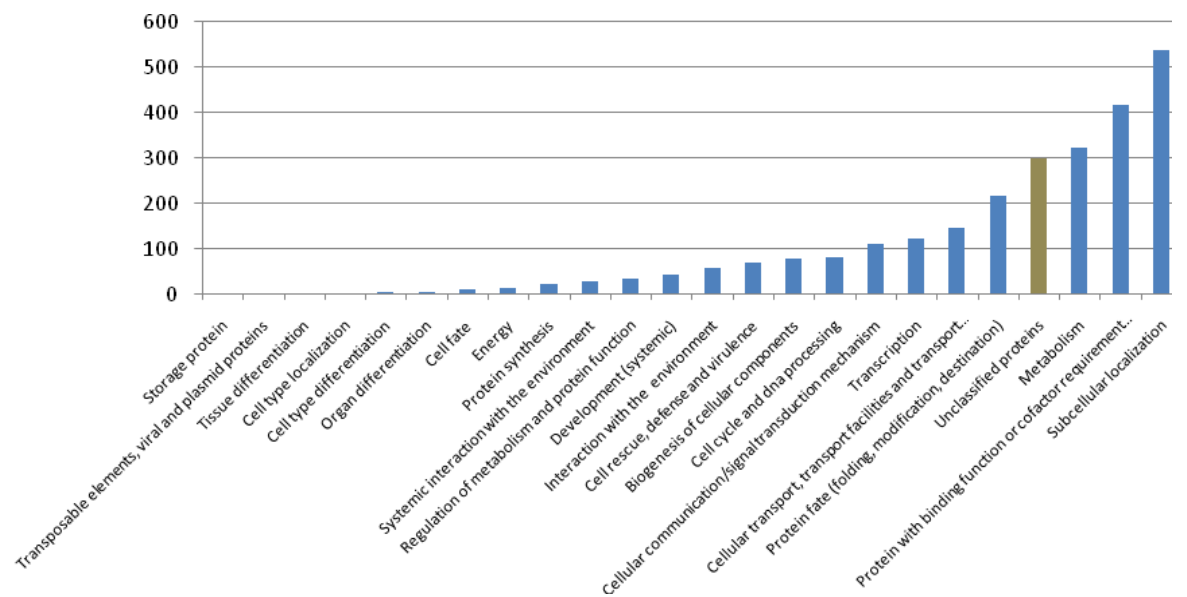

Figure 4. Genes pertaining to different metabolic pathways significantly down regulated under drought stress 


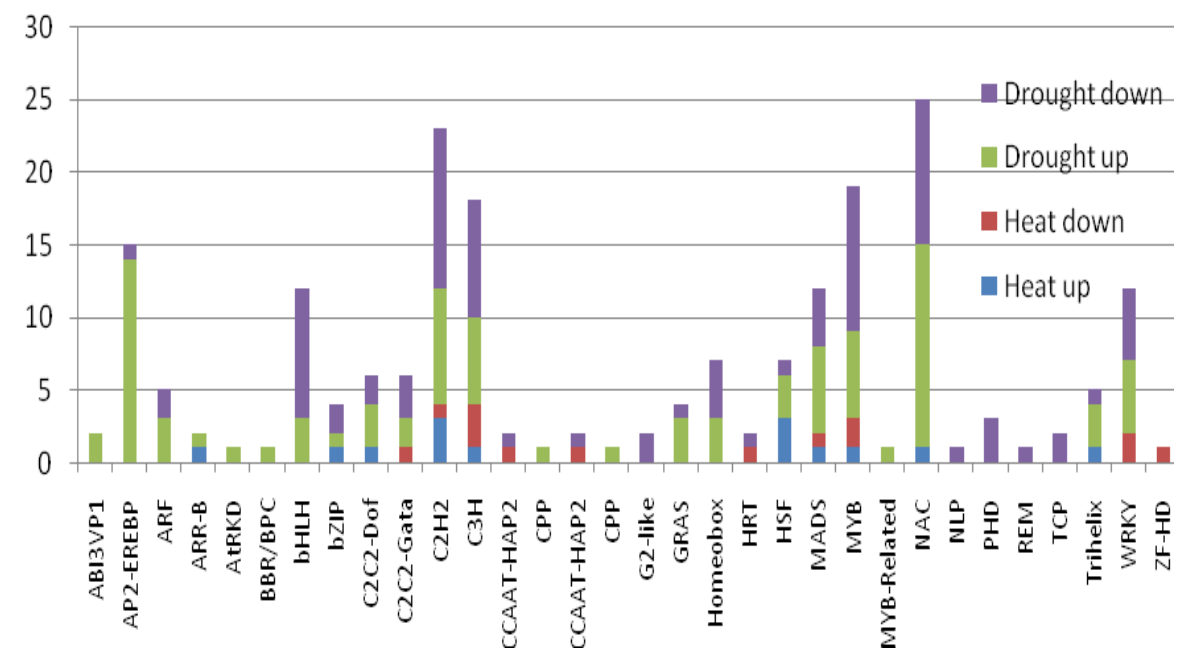

Figure 5. Transcription factor families represented under different stress conditions

\section{Discussion}

Drought and heat stress although have different effect on the transcript level of the cell, however many of the genes that are up or downregulated under the two kind of stress belong to the same metabolic process. It was observed that 95 genes were common among the upregulated genes in plants subjected to heat and drought stress. Similarly, a very close number of genes (101) were downregulated in plants subjected to the same kind of stresses. It points to the fact that plants have evolved more or less similar strategies for coping with different kinds of environmental stress factors. It has been reported earlier also in a study that subjected Arabidopsis plants simultaneously to heat and drought stress (Rhizsky et al., 2004). They found that the response of the plant is characterized by differential regulation of genes involved in respiration, photosynthesis and storage of metabolites. Further, it has been noted that the defense response is more or less similar and conserved among different plant species (Rhizsky et al., 2004). They also reported that similar genes were up or downregulated under combined influence of heat and drought stress. The overlap is an indication that the defense strategy of plants against drought and heat stress is coactivated in the same cell during combination of heat and drought stress. Similar results have been observed by Kreps et al., (2002), in defense response of plants for cold, drought and salinity.

One of the factors that enable the plants to cope up with changing environmental factors is its ability to modulate the response through the help of transcription factors (Chen et al., 2002). Present analysis finds $205 \mathrm{TFs}$ to be part of the response of plants to heat and drought stress. Of this AP2/EREBP family of TFs which was significantly upregualted under heat stress; it is known to play various roles in developmental processes and, in
Stress-related responses in plants (Sharoni et al., 2006). Similarly, NAC family constitutes one of the largest families of plant-specific transcription factors, and the family is present in a wide range of land plants (Olsen et al., 2005), and are reported to be capable of enhancing drought resistance and salt tolerance in rice plants ( $\mathrm{Hu}$ et al., 2006).

\section{References}

1. Abdeen, A., Jaimie, S., Brian, M., Transcriptome Analysis Reveals Absence of Unintended Effects in Drought-tolerant Transgenic Plants Overexpressing the Transcription Factor ABF3. BMC Genomics, 11(1), (2010): 69.

2. Charng, Y.Y., Liu, H.C., Liu, N.Y., Hsu, F.C. Ko, S.S., Arabidopsis Hsa32, a Novel Heat Shock Protein, Is Essential for Acquired Thermotolerance During Long Recovery After Acclimation. Plant Physiology , 140 (4), (2006):1297-1305.

3. Mizoguchi, M., T. Umezawa, K., Nakashima, S., Kidokoro, H., Takasaki, Y., Fujita, K.,YamaguchiShinozaki., Shinozaki, K., Two Closely Related Subclass II SnRK2 Protein Kinases Cooperatively Regulate Drought-Inducible Gene Expression. Plant and Cell Physiology, 51( 5), (2010):842-847.

4. Bowler, C., Fluhr, R., The role of calcium and activated oxygens as signals for controlling crosstolerance. Trends in Plant Science, 5(6), (2000): 241-246.

5. Chen, W., Provart, N. J., Glazebrook, J., Katagiri, F., Chang, H.-S., Eulgem, T., Mauch, F., Expression Profile Matrix of Arabidopsis Transcription Factor Genes Suggests Their Putative Functions in Response to Environmental Stresses. The Plant Cell Online, 14(3), (2002):559-574.

6. Dat, J., Vandenabeele, S., Vranová, E., Van Montagu, M., Inzé, D., Van Breusegem, F.. Dual action of the active oxygen species during plant stress responses. Cellular and Molecular Life Sciences: CMLS, 57(5), (2000):779-795. 
7. Hu, H., Dai, M., Yao, J., Xiao, B., Li, X., Zhang, Q. Xiong, L., Overexpressing a NAM, ATAF, and CUC (NAC) transcription factor enhances drought resistance and salt tolerance in rice. Proceedings of the National Academy of Sciences, 103(35), (2006):1298712992. doi:10.1073/pnas.0604882103

8. Kreps, J. A., Wu, Y., Chang, H.-S., Zhu, T., Wang, X. Harper, J. F., Transcriptome Changes for Arabidopsis in Response to Salt, Osmotic, and Cold Stress. Plant Physiology, 130(4), (2002): 2129-2141.

9. Mewes, H. W., Ruepp, A., Theis, F., Rattei, T., Walter, M., Frishman, D., Suhre, K.,MIPS: curated databases and comprehensive secondary data resources in 2010. Nucleic Acids Research, 39(Database), (2010):D220D224.

10. Mittler, R., Feng, X., Cohen, M., Post-Transcriptional Suppression of Cytosolic Ascorbate Peroxidase Expression during Pathogen-Induced Programmed Cell Death in Tobacco. The Plant Cell Online, 10(3), (1998):461-474

11. Olsen, A. N., Ernst, H. A., Leggio, L. L., Skriver, K., NAC transcription factors: structurally distinct, functionally diverse. Trends in Plant Science, 10(2), (2005): 79-87. doi:10.1016/j.tplants.2004.12.010.

12. Rizhsky, L., Liang, H., Shuman, J., Shulaev, V., Davletova, S., Mittler, R., When Defense Pathways Collide. The Response of Arabidopsis to a Combination of Drought and Heat Stress. Plant Physiology, 134(4), (2004): 1683-1696. doi:10.1104/pp.103.033431.
13. Seki, M., Narusaka, M., Ishida, J., Nanjo, T., Fujita, M., Oono, Y., Kamiya, A., Monitoring the expression profiles of 7000 Arabidopsis genes under drought, cold and high-salinity stresses using a full-length cDNA microarray. The Plant Journal, 31(3), (2002):279292. doi:10.1046/j.1365-313X.2002.01359.

14. Sharoni, A. M., Nuruzzaman, M., Satoh, K. Moumeni, A., Attia, K., Venuprasad, R., Serraj, R. Comparative transcriptome analysis of AP2/EREBP gene family under normal and hormone treatments, and under two drought stresses in NILs setup by Aday Selection and IR64. Molecular Genetics and Genomics: $\quad$ MGG, 287(1), (2012): 1-19. doi:10.1007/s00438-011-0659-3.

15. Xiong, L., Ishitani, M., Zhu, J.-K., Interaction of Osmotic Stress, Temperature, and Abscisic Acid in the Regulation of Gene Expression in Arabidopsis. Plant Physiology, 119(1), (1999):205-212.

16. Yilmaz, A., Mejia-Guerra, M. K., Kurz, K., Liang, X., Welch, L., Grotewold, E., AGRIS: the Arabidopsis Gene Regulatory Information Server, an update. Nucleic Acids Research, 39(Database issue), (2011): D1118-1122.

\section{Cite this article as:}

Murali Oleti. Effect of heat and drought stress on the expression of regulatory transcription factors and the genes involved in different metabolic pathways. Annals of Plant Sciences 7.1 (2018) pp. 1941-1945.

do http://dx.doi.org/10.21746/aps.2018.7.1.3 